\title{
Erratum to: Microvascular effects of glucagon-like peptide-1 receptor agonists in type 2 diabetes: a meta-analysis of randomized controlled trials
}

\author{
Ilaria Dicembrini ${ }^{1} \cdot$ Besmir Nreu $^{1} \cdot$ Alessia Scatena $^{2} \cdot$ Francesco Andreozzi $^{3}$. \\ Giorgio Sesti ${ }^{3} \cdot$ Edoardo Mannucci $^{1} \cdot$ Matteo Monami $^{1}{ }^{1}$
}

Published online: 19 September 2017

(C) Springer-Verlag Italia S.r.1. 2017

\section{Erratum to: Acta Diabetol \\ DOI 10.1007/s00592-017-1031-9}

In the manuscript, the events of nephropathy and retinopathy recorded for the LEADER trial (Marso et al. 2016) were erroneously imputed; the numbers reported in Table 1 for that trial refer to serious adverse events, and not to total events, as stated in Methods. The correct figures are 268 and 416 cases of nephropathy, and 106 and 92 cases of retinopathy in liraglutide and control arms, respectively.

As a consequence, the overall risk (MH-OR [95\% CI]) of nephropathy and retinopathy with GLP-1RA (vs all comparators) is 0.77 [0.67-0.88], $p<0.001$, and 0.99 [0.80-1.21], $p=0.90$, respectively. The analyses reported in Figs. 1 and 2 were also corrected and reported below, together with supplementary figures 3 and 5. Please note that liraglutide is associated with a significant reduction in the incidence of nephropathy, whereas for retinopathy the difference from placebo is no longer statistically significant.

The online version of the original article can be found under doi:10.1007/s00592-017-1031-9.

Matteo Monami

matteo.monami@unifi.it

Edoardo Mannucci

edoardo.mannucci@unifi.it

1 Diabetology, Azienda Ospedaliero Universitaria Careggi, University of Florence, Via delle Oblate 4, 50141 Florence, Italy

2 Diabetology Unit, Ospedale San Donato Arezzo, Arezzo, Italy

3 Department of Medical and Surgical Sciences, University Magna Graecia of Catanzaro, Catanzaro, Italy 


\begin{tabular}{|c|c|c|c|c|c|c|c|}
\hline \multirow[t]{2}{*}{$\underline{\text { Study name }}$} & \multicolumn{5}{|c|}{ Statistics for each study } & \multicolumn{2}{|c|}{ Events / Total } \\
\hline & $\begin{array}{l}\text { MH odds } \\
\text { ratio }\end{array}$ & $\begin{array}{c}\text { Lower } \\
\text { limit }\end{array}$ & $\begin{array}{c}\text { Upper } \\
\text { limit }\end{array}$ & Z-Value & p-Value & SGLT2i & Comparator \\
\hline Diamant 2014 (26) & 7,544 & 0,388 & 146,796 & 1,334 & 0,182 & $3 / 247$ & $0 / 263$ \\
\hline Zinman $2009(77)$ & 5,508 & 0,303 & 100,173 & 1,153 & 0,249 & $5 / 355$ & $0 / 175$ \\
\hline Pinget 2013 (109) & 4,549 & 0,243 & 85,016 & 1,014 & 0,311 & $4 / 323$ & $0 / 161$ \\
\hline Reusch $2014(7)$ & 3,647 & 0,745 & 17,850 & 1,597 & 0,110 & $7 / 150$ & $2 / 151$ \\
\hline Zang $2016(87)$ & 3,033 & 0,123 & 74,937 & 0,678 & 0,498 & $1 / 183$ & $0 / 184$ \\
\hline Gallwitz 2012 (31) & 2,988 & 0,121 & 73,522 & 0,670 & 0,503 & $1 / 490$ & $0 / 487$ \\
\hline Pratley 2011 (69) & 2,744 & 0,603 & 12,487 & 1,305 & 0,192 & $11 / 446$ & $2 / 219$ \\
\hline Riddle 2013 (104) & 2,565 & 0,122 & 53,735 & 0,607 & 0,544 & $2 / 328$ & $0 / 167$ \\
\hline Nauck 2016 (8) & 2,564 & 0,296 & 22,245 & 0,854 & 0,393 & $5 / 200$ & $1 / 101$ \\
\hline Marso 2016 (113) & 1,748 & 1,100 & 2,776 & 2,365 & 0,018 & $50 / 1648$ & $29 / 1649$ \\
\hline Ahrén 2013 (106) & 1,676 & 0,080 & 35,094 & 0,333 & 0,739 & $2 / 510$ & $0 / 170$ \\
\hline NCT01644500 & 1,521 & 0,062 & 37,461 & 0,257 & 0,798 & $1 / 536$ & $0 / 271$ \\
\hline Garber $2011(76)$ & 1,498 & 0,061 & 36,918 & 0,247 & 0,805 & $1 / 498$ & $0 / 248$ \\
\hline Bolli 2014 (102) & 1,498 & 0,061 & 36,970 & 0,247 & 0,805 & $1 / 322$ & $0 / 160$ \\
\hline Rosenstock 2014 (111) & 1,493 & 0,061 & 36,776 & 0,245 & 0,806 & $1 / 574$ & $0 / 285$ \\
\hline Ahrén 2014 (1) & 1,452 & 0,737 & 2,862 & 1,077 & 0,281 & $14 / 302$ & $23 / 710$ \\
\hline Araki 2015 (14) & 1,333 & 0,294 & 6,044 & 0,373 & 0,709 & $4 / 181$ & $3 / 180$ \\
\hline Miyagawa 2015 (13) & 1,255 & 0,144 & 10,913 & 0,205 & 0,837 & $5 / 280$ & $1 / 70$ \\
\hline Kaku 2010 (83) & 1,157 & 0,401 & 3,341 & 0,270 & 0,787 & $8 / 88$ & $7 / 88$ \\
\hline Marso $2016(79)$ & 1,157 & 0,872 & 1,534 & 1,011 & 0,312 & $106 / 4668$ & $92 / 4672$ \\
\hline Leiter 2014 (6) & 1,082 & 0,468 & 2,500 & 0,184 & 0,854 & $12 / 249$ & $11 / 246$ \\
\hline Weissman 2014 (9) & 0,905 & 0,414 & 1,977 & $-0,250$ & 0,802 & $19 / 504$ & $10 / 241$ \\
\hline Weinstock 2015 (18) & 0,879 & 0,036 & 21,682 & $-0,079$ & 0,937 & $1 / 606$ & $0 / 177$ \\
\hline Rosenstock 2014 (2) & 0,869 & 0,493 & 1,531 & $-0,487$ & 0,627 & $25 / 285$ & $28 / 281$ \\
\hline Seino 2010 (82) & 0,775 & 0,341 & 1,757 & $-0,611$ & 0,541 & $16 / 268$ & $10 / 132$ \\
\hline Marre $2009(74)$ & 0,764 & 0,354 & 1,649 & $-0,687$ & 0,492 & $17 / 695$ & $11 / 346$ \\
\hline NCT01733758 & 0,751 & 0,030 & 18,619 & $-0,175$ & 0,861 & $1 / 310$ & $0 / 77$ \\
\hline Home $2015(10)$ & 0,713 & 0,328 & 1,547 & $-0,856$ & 0,392 & $10 / 271$ & $20 / 392$ \\
\hline Russell-Jones 2009 (78) & 0,640 & 0,164 & 2,501 & $-0,642$ & 0,521 & $3 / 230$ & $7 / 346$ \\
\hline Nauck 2009 (75) & 0,432 & 0,257 & 0,725 & $-3,171$ & 0,002 & $29 / 724$ & $32 / 363$ \\
\hline Seino 2012 (103) & 0,338 & 0,014 & 8,353 & $-0,663$ & 0,507 & $0 / 154$ & $1 / 157$ \\
\hline Mathieu $2014(70)$ & 0,333 & 0,013 & 8,294 & $-0,670$ & 0,503 & $0 / 88$ & $1 / 89$ \\
\hline Pfeffer 2015 (101) & 0,333 & 0,067 & 1,651 & $-1,346$ & 0,178 & $2 / 3034$ & $6 / 3034$ \\
\hline Diamant 2012 (64) & 0,318 & 0,013 & 7,838 & $-0,701$ & 0,483 & $0 / 233$ & $1 / 223$ \\
\hline Elonde 2015 (11) & 0,166 & 0,017 & 1,606 & $-1,550$ & 0,121 & $1 / 588$ & $3 / 296$ \\
\hline NCT01648582 & 0,164 & 0,007 & 4,030 & $-1,107$ & 0,268 & $0 / 516$ & $1 / 254$ \\
\hline NCT00614120 & 0,163 & 0,030 & 0,896 & $-2,086$ & 0,037 & $2 / 698$ & $4 / 231$ \\
\hline & 0,987 & 0,803 & 1,213 & $-0,127$ & 0,899 & & \\
\hline
\end{tabular}

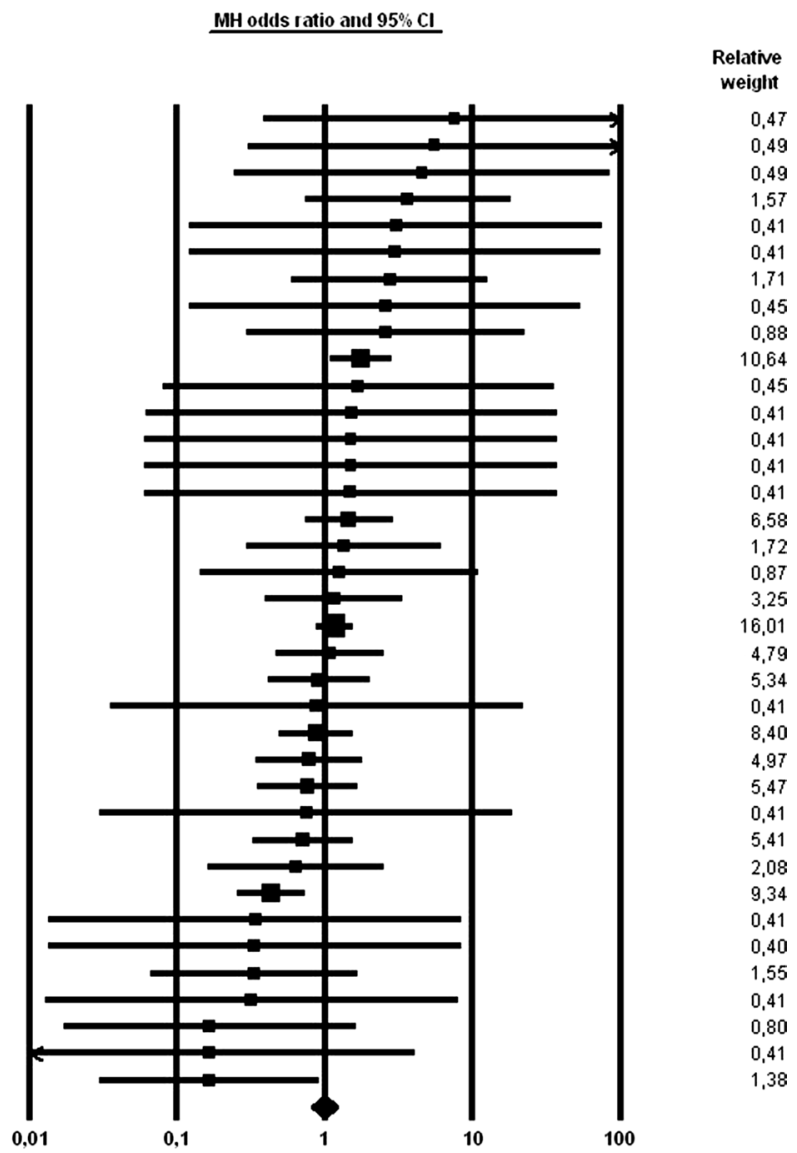

Favours GLP-1RA Favours Comparators

Suppl. Figure 3 Mantel-Haenzel odds ratio for retinopathy with 95\% CI. GLP1-RA: Glucagon-Like Peptide 1 Receptor Agonists 


\begin{tabular}{|c|c|c|c|c|c|c|c|}
\hline \multirow[t]{2}{*}{ Study name } & \multicolumn{5}{|c|}{ Statistics for each study } & \multicolumn{2}{|c|}{ Events / Total } \\
\hline & $\begin{array}{l}\text { MH odds } \\
\text { ratio }\end{array}$ & $\begin{array}{l}\text { Lower } \\
\text { limit }\end{array}$ & $\begin{array}{l}\text { Upper } \\
\text { limit }\end{array}$ & Z-Value & $p$-Value & SGLT2i & Comparator \\
\hline Ahrén 2014 (1) & 7,070 & 0,2871 & 174,034 & 1,197 & 0,231 & $1 / 302$ & $0 / 710$ \\
\hline Rosenstock 2014 (2) & 0,327 & 0,013 & 8,073 & $-0,683$ & 0,495 & $0 / 285$ & $1 / 281$ \\
\hline Leiter 2014 (6) & 0,987 & 0,509 & 1.913 & $-0,039$ & 0,969 & $19 / 249$ & $19 / 246$ \\
\hline Reusch 2014 (7) & 3,040 & 0,123 & 75,224 & 0,679 & 0,497 & $1 / 150$ & $0 / 151$ \\
\hline NCT01648582 & 0,326 & 0,054 & 1,961 & $-1,225$ & 0,221 & $2 / 516$ & $3 / 254$ \\
\hline Miyagawa 2015 (13) & 1,000 & 0,110 & 9,090 & 0,000 & 1,000 & $4 / 280$ & $1 / 70$ \\
\hline Araki 2015 (14) & 2,000 & 0,180 & 22,254 & 0,564 & 0,573 & $2 / 181$ & $1 / 180$ \\
\hline Giorgino 2015 & 0,160 & 0,006 & 3,936 & $-1,122$ & 0,262 & $0 / 545$ & $1 / 262$ \\
\hline Gallwitz 2012 (31) & 4,990 & 0,2391 & 104,203 & 1,037 & 0,300 & $2 / 490$ & $0 / 487$ \\
\hline Marre $2009(74)$ & 1,496 & 0,155 & 14,432 & 0,348 & 0,728 & $3 / 695$ & $1 / 346$ \\
\hline Nauck 2009 (75) & 0,833 & 0,300 & 2,311 & $-0,350$ & 0,726 & $10 / 724$ & $6 / 363$ \\
\hline Zinman $2009(77)$ & 0,069 & 0,004 & 1.349 & $-1,762$ & 0,078 & $0 / 355$ & $3 / 175$ \\
\hline Russell-Jones 2009 (78) & 3) 0,500 & 0,020 & 12,318 & $-0,424$ & 0,671 & $0 / 230$ & $1 / 346$ \\
\hline Marso $2016(79)$ & 0,784 & 0,664 & 0,925 & $-2,884$ & 0,004 & $268 / 4668$ & $337 / 4672$ \\
\hline Davies 2016 & 0,875 & 0,328 & 2,339 & $-0,265$ & 0,791 & $8 / 140$ & $9 / 139$ \\
\hline NCTO0614120 & 0,996 & 0,040 & 24,526 & $-0,003$ & 0,998 & $1 / 698$ & $0 / 231$ \\
\hline Zang 2016 (87) & 3,033 & 0,123 & 74,937 & 0,678 & 0,498 & $1 / 183$ & $0 / 184$ \\
\hline Davies 2015 & 3,029 & 0,123 & 74.765 & 0,677 & 0,498 & $1 / 211$ & $0 / 212$ \\
\hline NCT00976937 & 0,338 & 0,014 & 8,348 & $-0,664$ & 0,507 & $0 / 158$ & $1 / 161$ \\
\hline Pfeffer 2015 (101) & 1,000 & 0,350 & 2,854 & 0,000 & 1,000 & $7 / 3034$ & $7 / 3034$ \\
\hline Riddle 2013 (104) & 0,169 & 0,007 & 4,170 & $-1,087$ & 0,277 & $0 / 328$ & $1 / 167$ \\
\hline Yu Pan 2014 & 0,989 & 0,446 & 2.192 & $-0,027$ & 0,978 & $13 / 196$ & $13 / 194$ \\
\hline Marso 2016 (113) & 0,606 & 0,438 & 0.838 & $-3,030$ & 0,002 & $62 / 1648$ & $100 / 1649$ \\
\hline & 0,769 & 0,671 & 0,880 & $-3,815$ & 0,000 & & \\
\hline
\end{tabular}

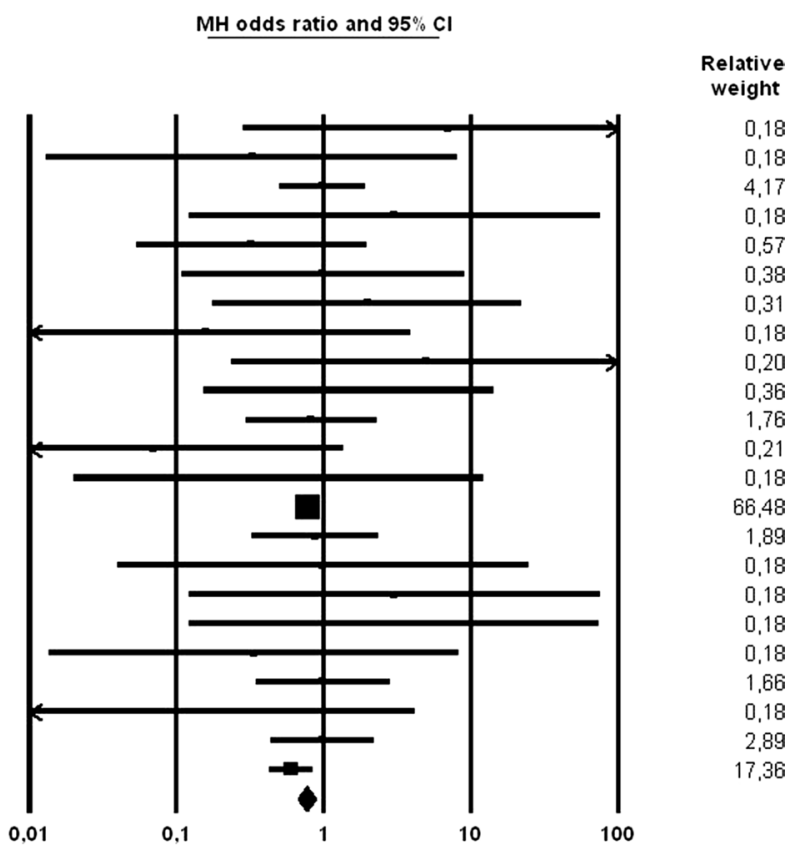

Favours GLP-1RA Favours Comparators

Suppl. Figure 5 Mantel-Haenzel odds ratio for nephropathy with 95\% CI. GLP1-RA: Glucagon-Like Peptide 1 Receptor Agonists
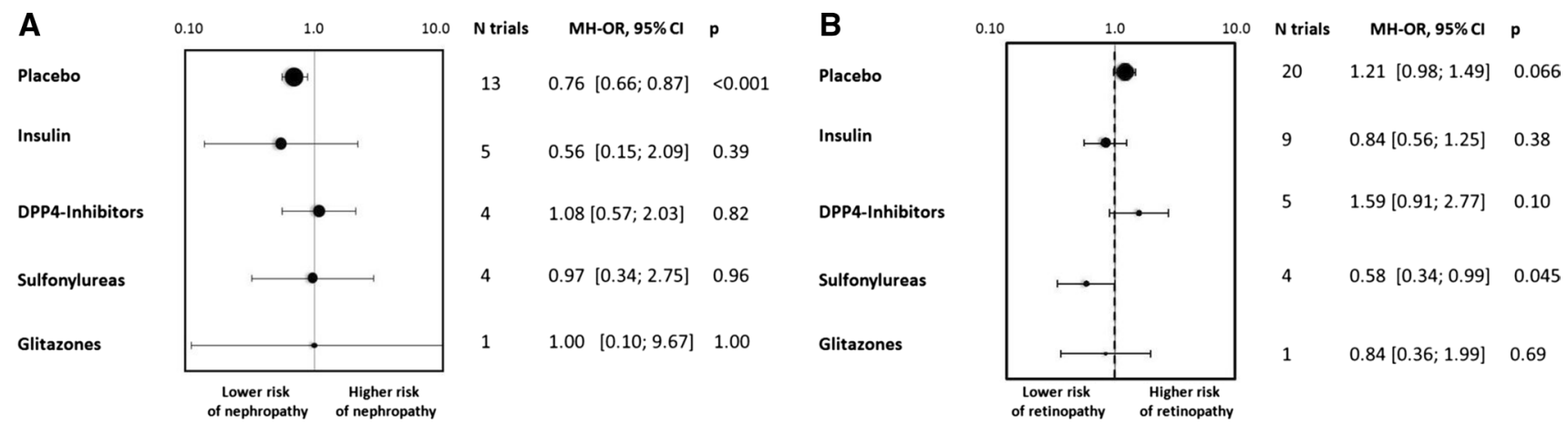

Fig. 1 MH-OR with 95\% CI for GLP-1 receptor agonists versus different comparators for nephropathy (a) and retinopathy (b)
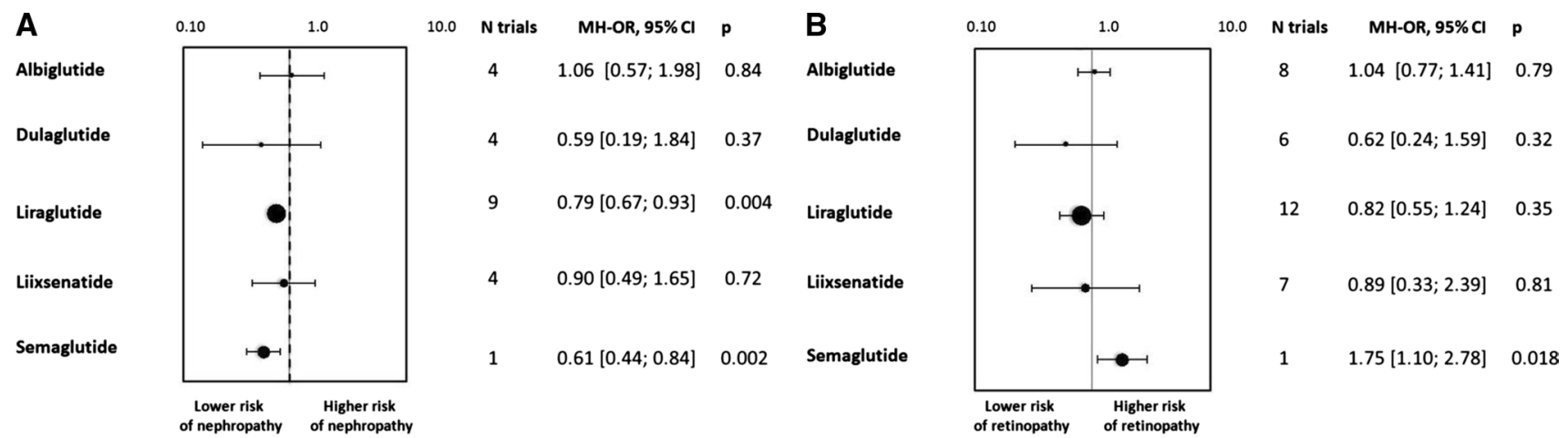

Fig. 2 MH-OR with 95\% CI for different GLP-1 receptor agonists versus other comparators for nephropathy (a) and retinopathy (b) 